7th International Workshop on Astronomy and

Relativistic Astrophysics (IWARA 2016)

International Journal of Modern Physics: Conference Series

Vol. 45 (2017) 1760049 (5 pages)

(C) The Author(s)

DOI: $10.1142 / S 2010194517600497$

\title{
Ansatz for Dense Matter Equation of State
}

\author{
Marcelo D. Alloy \\ Departamento de Ciências Exatas e Educação \\ Universidade Federal de Santa Catarina (UFSC) \\ Blumenau, SC, CEP 89.065-300, Brazil \\ marcelo.alloy@ufsc.br \\ Débora P. Menezes \\ Departamento de Física \\ Universidade Federal de Santa Catarina (UFSC) \\ Florianópolis, SC, CP 476, CEP 88.040-900, Brazil \\ debora.p.m@ufsc.br \\ Manuel Malheiro \\ Instituto Tecnológico de Aeronáutica (ITA) \\ São José dos Campos, SP, CEP 12228-900, Brazil \\ malheiro@ita.br
}

Published 15 August 2017

\begin{abstract}
The aim of the present work is to try to find an equation of state (EoS) directly from the solution of the Tolman-Oppenheimer-Volkoff (TOV) equations subject to known observational constraints to the maximum mass and corresponding radius and baryonic mass. Hence, instead of solving the TOV equations with an EoS that enters as input, we obtain an EoS as output.
\end{abstract}

Keywords: Equation of State, Neutron Stars, Compact Stars.

PACS numbers: 26.60.-c, 97.60.Jd, 11.10.-z

\section{Introduction and Formalism}

The correct description of neutron star (NS) properties depends on two ingredients: the use of appropriate equations of state and the solution of the OppenheimerVolkoff $(\mathrm{OV})^{1}$ or Tolman-Oppenheimer-Volkoff (TOV) ${ }^{1,2}$ equations, as many people often call them ${ }^{3}$. In the last decade two massive pulsars were observed, the PSR J1614-2230 with mass $1.97 \pm 0.04 M_{\odot}$ (see Ref. ${ }^{4}$ ) and the PSR J0348+0432 with

This is an Open Access article published by World Scientific Publishing Company. It is distributed under the terms of the Creative Commons Attribution 4.0 (CC-BY) License. Further distribution of this work is permitted, provided the original work is properly cited. 
mass $2.01 \pm 0.04 M_{\odot}$ (see Ref. ${ }^{5}$ ) and their existence made theoreticians revisit many of the existing models so that massive stars could be described. Neutron stars radii are not so well known, but we expect that the future X-ray mission ATHENA to be launched in 2018, combined with accurate distance measurements made by GAIA, will give us information on simultaneously obtained measurements of masses and radii of several dozens of neutron stars. Another important quantity related to the equation of state of dense matter is the sound velocity, which should be limited to $v_{s}^{2}=1 / 3$ (in natural units) ${ }^{6}$. The above considerations have motivated us to look for an expression for the sound velocity and consequently to the pressure as a function of the energy density with a non-linear dependence on the central energy density that would be the solution of the Tolman-Oppenheimer-Volkoff (TOV) equations ${ }^{2}$. A similar approach was used in Ref. ${ }^{7}$, where the authors have obtained EoS for compact stars by solving a constraint equation derived from the bulk equation of motion together with the TOV equations.

The TOV equations consist of a set of differential equations developed to describe static, spheric and compact stars in hydrostatic equilibrium, while taking into account special and general relativistic effects and are given by:

$$
\begin{aligned}
\frac{d p}{d r} & =-\frac{(\epsilon(r)+p(r))\left(M(r)+4 \pi p(r) r^{3}\right)}{r^{2}-2 M(r) r} \\
\frac{d M}{d r} & =4 \pi \epsilon(r) r^{2} \\
\frac{d M_{B}}{d r} & =\frac{4 \pi r^{2} m_{n} \rho(r)}{(1-2 M(r) / r)^{1 / 2}},
\end{aligned}
$$

where $\epsilon$ is the energy density, $p$ is the pressure, $M$ the gravitational mass, $M_{B}$ is the baryonic mass, $\rho$ is the baryonic density, $r$ the radial coordinate and $m_{n}$ is the nucleon mass. In this paper, we propose a mathematical formulation as an ansatz for the EoS of nuclear matter given by:

$$
p=A \epsilon^{C}+B \epsilon
$$

where $A, B$ and $C$ are positive parameters which are varied in such a way that they satisfy the following conditions: $\epsilon(r=0)=12 \mathrm{fm}^{-4}$ and $\epsilon(r=R)=0.25 \mathrm{fm}^{-4}$. We then restrict the solutions so that the maximum masses and related radii obtained for a family of stars lie in the range $1.49<M_{\max }\left(M_{\odot}\right)<2.71$ and $10.0<R(\mathrm{~km})<15.01$, with the extra condition that the maximum baryonic mass is such that $M_{\max }<M_{B, \max }<2 M_{\max }$. The baryonic number density is obtained directly from

$$
\frac{\partial \epsilon}{\partial \rho}=\frac{\epsilon+p}{\rho},
$$

and hence, obeys the thermodynamic consistency condition. In the study of neutron stars, the maximum mass and the sound velocity are important ingredients in the 
identification of appropriate nuclear and hadron physics models. The sound velocity, obtained from eq.(2) is trivially given by

$$
v_{s}^{2}=\frac{d p}{d \epsilon}=A C \epsilon^{C-1}+B .
$$

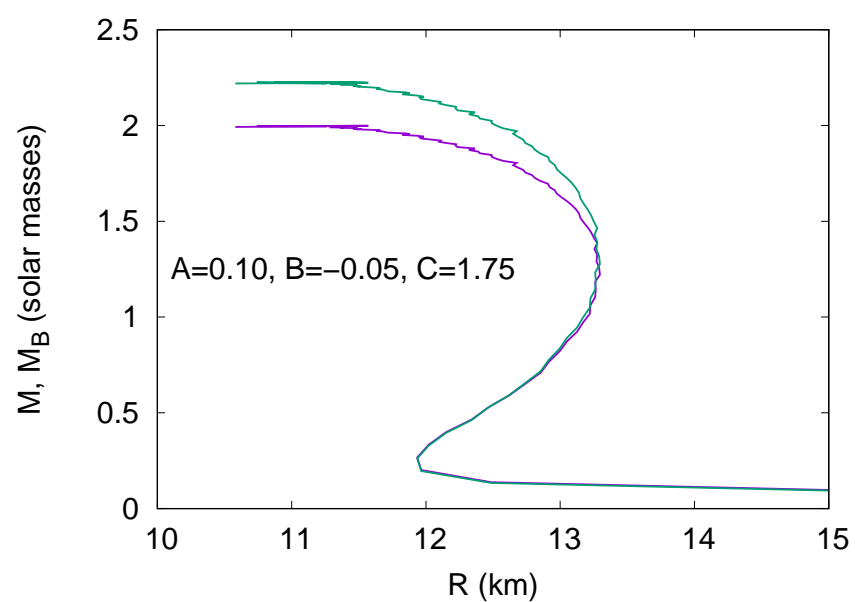

Fig. 1. Mass-radius curves with the inclusion of the BPS for two different EoS. The curves with the higher maxima refer to the baryonic masses while the ones with the lower maxima refer to the gravitational masses.

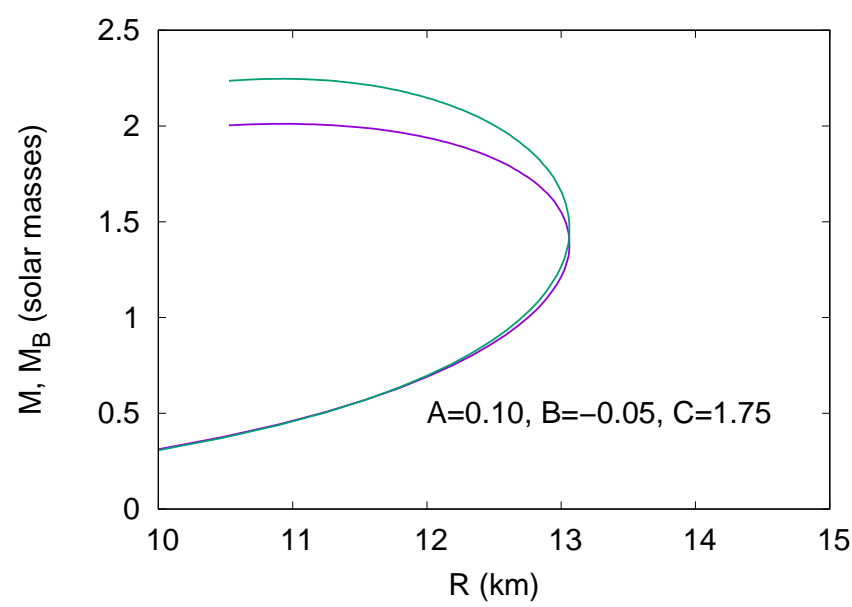

Fig. 2. Mass-radius curves without the inclusion of the BPS for two different EoS. The curves with the higher maxima refer to the baryonic masses while the ones with the lower maxima refer to the gravitational masses. 


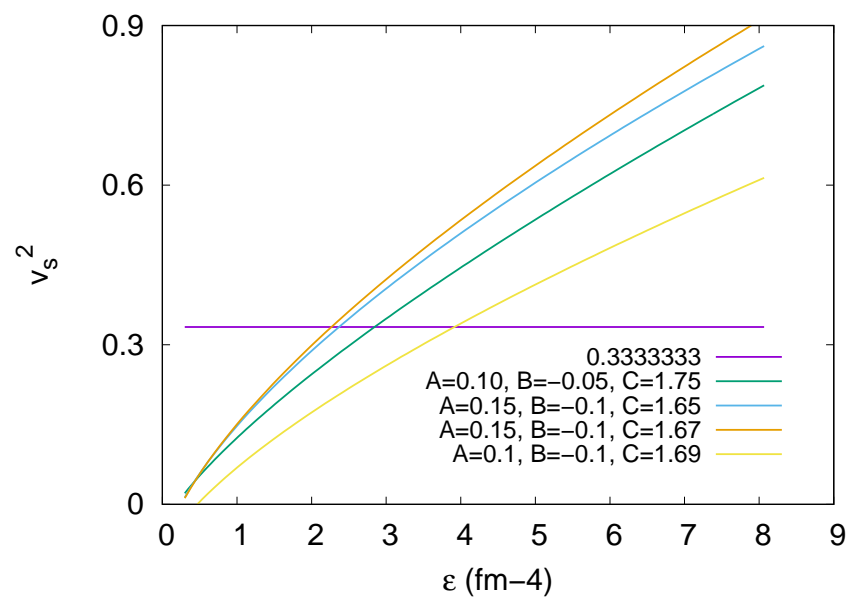

Fig. 3. Squared sound velocity for different EoS.

Table 1. Maximum gravitational and baryonic masses, respective radii and central baryonic densities.

\begin{tabular}{cccccccc}
\hline $\mathrm{A}$ & $\mathrm{B}$ & $\mathrm{C}$ & $\begin{array}{c}M_{\max } \\
\left(M_{\odot}\right)\end{array}$ & $\begin{array}{c}M_{B_{\max }} \\
\left(M_{\odot}\right)\end{array}$ & $\begin{array}{c}\mathrm{R} \\
(\mathrm{km})\end{array}$ & $v_{s c}^{2}$ & $\begin{array}{c}\epsilon_{c} \\
\left(\mathrm{fm}^{-4}\right)\end{array}$ \\
\hline 0.10 & -0.1 & 1.69 & 1.67 & 2.00 & 9.18 & 0.61 & 8.02 \\
0.10 & -0.1 & 1.71 & 1.71 & 2.06 & 9.24 & 0.65 & 8.02 \\
0.10 & -0.1 & 1.73 & 1.76 & 2.12 & 9.31 & 0.69 & 8.02 \\
0.10 & -0.1 & 1.75 & 1.80 & 2.18 & 9.37 & 0.73 & 8.02 \\
0.10 & -0.05 & 1.65 & 1.84 & 2.01 & 10.71 & 0.54 & 7.18 \\
0.10 & -0.05 & 1.67 & 1.87 & 2.06 & 10.77 & 0.57 & 7.06 \\
0.10 & -0.05 & 1.69 & 1.91 & 2.11 & 10.80 & 0.60 & 7.02 \\
0.10 & -0.05 & 1.71 & 1.94 & 2.15 & 10.87 & 0.62 & 6.90 \\
0.10 & -0.05 & 1.73 & 1.98 & 2.20 & 10.87 & 0.66 & 6.95 \\
0.10 & -0.05 & 1.75 & 2.01 & 2.25 & 10.93 & 0.69 & 6.82 \\
0.15 & -0.1 & 1.65 & 2.19 & 2.53 & 11.67 & 0.68 & 5.83 \\
0.15 & -0.1 & 1.67 & 2.23 & 2.58 & 11.77 & 0.70 & 5.69 \\
0.15 & -0.1 & 1.69 & 2.27 & 2.64 & 11.82 & 0.74 & 5.64 \\
0.15 & -0.1 & 1.71 & 2.30 & 2.69 & 11.87 & 0.77 & 5.58 \\
0.15 & -0.1 & 1.73 & 2.34 & 2.75 & 11.92 & 0.80 & 5.53 \\
0.15 & -0.1 & 1.75 & 2.37 & 2.80 & 12.01 & 0.83 & 5.38 \\
\hline
\end{tabular}

\section{Results and Conclusions}

To obtain the parameters that satisfy all the above constraints, we vary $A$ from 0.05 to 0.5 in steps of $0.05, B$ from -0.5 ato 0.0 in steps of 0.05 and $C$ from 1.65 to 1.75 in steps of 0.02 . The baryonic density range goes from $0.25 \mathrm{fm}^{-3}$ to $8.0 \mathrm{fm}^{-3}$. Within the above mentioned ranges, all possible results for the free parameters are displayed in Table 1. If one wants these EoS to describe hadronic stars, a crust describing very low density matter is expected to be present. For this purpose the BPS (see Ref. ${ }^{8}$ ) EoS has to be added to the EoS obtained from our prescription and 
the mass radius relations have to be reobtained from the TOV equations, resulting in the mass-radius curves displayed in Fig. 1 and Fig. 2. One can also see from Table 1 and Fig. 3 that the squared sound velocity at the center of the star always exceeds $1 / 3$, a conclusion also reached for relativistic mean field (RMF) models ${ }^{9}$ and foreseen in Ref. ${ }^{6}$.

In this work a possible mathematical form of an equation of state was obtained from the Tolman-Oppenheimer-Volkoff equations. The parameter values were obtained so that the EoS reproduces observational constraints and obeys casualty. We have verified that, if no other restriction is imposed, the sound velocity is usually larger than what is predicted by QCD. Nevertheless, at this high densities, a transition to another form of matter could exist and at the transition point, the sound velocity could decrease before increasing again.

\section{Acknowledgments}

D. P. M. acknowledges support by CNPq (grant 300602/2009-0).

\section{References}

1. J. R. Oppenheimer and G. M. Volkoff, Phys. Rev. 55, 374 (1939).

2. R. C. Tolman, Phys. Rev. 55, 364 (1939).

3. I. Semiz, arXiv:1610.03049 (2016).

4. P. B. Demorest, T. Pennucci, S. M. Ransom, M. S. E. Roberts, and J. W. T. Hessels, Nature 467, 1081 (2010).

5. J. Antoniadis, et al., Science 340, 1233232 (2013) .

6. P. Bedaque and A. W. Steiner, Phys. Rev. Lett. 114, 031103 (2015).

7. K. K. Kim, Y. Kim, and I. J. Shin, arXiv:1206.4421[hep-ph] (2012).

8. G. Baym, C. Pethick, and P. Sutherland, Astrophys. J. 170, 299 (1971).

9. M. Dutra, O. Lourenço, and D. P. Menezes, Phys. Rev. C 94(E), 049901 (2016); Phys. Rev. C 93, 025806 (2016). 